Registration, Reporting, and Replication in Anorexia Nervosa

\title{
Registration, Reporting, and Replication in Clinical Trials: The Case of
}

\section{Anorexia Nervosa}

Stuart B. Murray, Ph.D. ${ }^{a}$, Emilio J. Compte, Ph.D. ${ }^{\mathrm{a}, \mathrm{b}, \mathrm{c}}$, Daniel S. Quintana, Ph.D. ${ }^{\mathrm{d}}$,

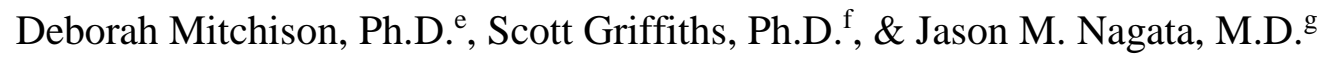

\author{
a Department of Psychiatry, University of California, San Francisco, San Francisco, CA, USA \\ ${ }^{\mathrm{b}}$ School of Human and Behavioral Sciences, Favaloro University, Buenos Aires, Argentina \\ ${ }^{\mathrm{c}}$ Eating Disorders Team, Fundación Foro, Buenos Aires, Argentina \\ ${ }^{\mathrm{d}}$ NORMENT, KG Jebsen Centre for Psychosis Research, Division of Mental Health and \\ Addiction, University of Oslo, and Oslo University Hospital, Oslo, Norway \\ ${ }^{\mathrm{e}}$ Translational Health Research Institute, Western Sydney University, Sydney, NSW, \\ Australia \\ ${ }^{\mathrm{f}}$ Department of Psychology, Macquarie University, Sydney, NSW, Australia \\ ${ }^{g}$ Melbourne School of Psychological Sciences, University of Melbourne, Melbourne, VIC, \\ Australia \\ ${ }^{\text {h }}$ Department of Pediatrics, University of California, San Francisco, San Francisco, CA, USA
}

Correspondence: Dr. Stuart B. Murray, Department of Psychiatry, University of California, San Francisco, 401 Parnassus, San Francisco, CA, 94143, Tel: +61 420838734 Email: stuart.murray@ucsf.edu; drstuartmurray@gmail.com

Keywords: Anorexia nervosa; clinical trials; methodological bias; ClinicalTrials.gov

This is the author manuscript accepted for publication and has undergone full peer review but has not been through the copyediting, typesetting, pagination and proofreading process, which may lead to differences between this version and the Version of Record. Please cite this article as doi: $10.1002 /$ eat.22907

This article is protected by copyright. All rights reserved. 
Word Count: 2,161

Abstract Word Count: 185

\section{RUNNING HEAD: REGISTRATION, REPORTING, AND REPLICATION IN ANOREXIA NERVOSA}

\section{Abstract}

Objective: Treatment outcomes for anorexia nervosa (AN) remain modest, and recent research suggests that clinical trials may be of limited methodological quality. With increasing evidence illustrating the irreproducibility of psychological research, no research to date has systematically examined the cumulative effect of bias in research relating to the treatment of AN.

Method: We identified all AN trials listed on ClinicalTrials.gov between 2000-2018 and examined rates of (i) the non-completion of clinical trials, the (ii) non-publication of trials once listed as completed, (iii) the non- prospective registration of clinical trials, and (iv) the non-replication of findings.

Results: We note that of 201 trials listed on ClinicalTrials.gov, only 101 have been completed, and of those, only 41 have been published. Moreover, of these 41 published trials, only 8 demonstrated evidence of prospective trial registration, and only 7 have had their primary findings replicated in other studies.

Discussion: These results illustrate the profound cumulative effect of methodological bias in registered trials for AN, which may have a significant impact both on what appears in the current evidence base, and on the reproducibility of studies comprising this evidence base.

This article is protected by copyright. All rights reserved. 


\section{Registration, Reporting, and Replication in Clinical Trials: The Case of Anorexia Nervosa}

Evidence-based treatment is the bedrock of clinical practice, and importantly, it is entirely dependent upon the quality of trials upon which the evidence base rests. With growing awareness of the irreproducibility of psychiatric and psychological research (Munafo et al., 2017), the discipline of “meta science” has emerged, whose goal is to evaluate and improve upon scientific practices. In a scientific climate that incentivizes innovation and novel findings, selective reporting is prevalent (Mathieu et al., 2009), and failed attempts to replicate findings indicate that false positive results and inflated effect sizes are prevalent (Camerer et al., 2018). Further, trials demonstrating non-significant findings appear (i) less likely to be published, (ii) less likely to completely report outcomes, and (iii) less likely to be cited (de Vries et al., 2018). As a result, the published literature may indicate stronger evidence than what actually exists, and 'negative' results may disappear from the evidence base.

The accumulation of these biases may have profound effects on the reported efficacy of treatments, and upon what constitutes an evidence-based treatment. In the

This article is protected by copyright. All rights reserved. 
case of anorexia nervosa (AN) where approximately two-thirds of those treated with specialized treatments do not remit from symptoms (Watson \& Bulik, 2013), and more than 50\% still meet diagnostic criteria two decades after illness onset (Fichter et al., 2017), careful analysis of the existing evidence base is critical. Recent metaanalyses assessing treatment outcomes in AN noted an elevated risk of bias across RCTs for AN, characterized by random sequence generation bias, allocation concealment bias, attrition bias, and reporting bias in up to $60 \%$ of trials (Murray et al., 2019; Zeeck et al., 2018). Additional analyses of trial registration and replications may further inform the methodological quality and utility of trials.

Good scientific conduct relating to clinical trials stipulates that protocols, hypotheses, primary outcomes and planned analyses should be prospectively declared and registered prior to the collection and analysis of data (Scott et al., 2015). When considering how various analytic approaches to address one research question with one dataset can yield widely varying results and effect sizes (Silberzahn et al., 2018), the non-prospective registration of trial protocol may permit posthoc selection of the analytic methods which best support study hypotheses. Indeed, many published findings in the medical literature stem from reports where secondary outcomes are reported as primary outcomes (when the original primary outcomes yield no significant results), or where posthoc analyses are reported as a priori, and the prospective registration of trial protocols is therefore crucial to the integrity and reproducibility of scientific findings (Munafo et al., 2017). As such, journals belonging to the International Committee of Medical Journals Editors (ICMJE) have 
now begun requesting evidence of prospective registration of clinical trials prior to their publication (De Angelis et al., 2004), although compliance with these recommendations remains an ongoing concern (Anderson et al., 2015).

Good scientific conduct also stipulates that upon completion or termination of clinical trials, deidentified and safeguarded research data ought to be openly, transparently and publicly shared (Walsh et al., 2018). In fact, in response to the growing awareness of publication bias in the biomedical literature (i.e., journals not publishing findings with non-significant findings, or researchers not publishing findings discordant with study hypotheses), and the withholding of potentially harmful data from public consumption (Turner et al., 2008), clinical trial registries now mandate the timely reporting of summary data upon completion or termination of all clinical trials, irrespective of whether findings are published in peer-reviewed journals. For instance, the Food and Drug Administration Amendments Act mandates that all clinical trial results in the United States be reported in publicly available trial registries within 1 year of study completion or termination. Not doing so, it is argued, may result in the disappearance of non-favorable results from the literature, which markedly skews the evidence base.

A third and crucial component of the scientific enterprise, specifically in the context of clinical trials, relates to the replication of study findings. Accepting findings from individual studies as scientific truths, prior to their replication by independent researchers, is vulnerable to false positives. A recent survey revealed that more than $70 \%$ of researchers have failed to replicate other researchers' findings, and 
more than half failed to replicate their own findings (Baker, 2016). Even attempts to replicate study results in samples up to five times larger than those of original trials yields replication of results in approximately $60 \%$ studies, albeit with effect sizes approximately 50\% of those reported in original trials (Camerer et al., 2018). This is especially salient in the context of clinical trials for AN, where sample sizes have historically been small and underpowered, and spurious findings may be more likely. Here, we aim to distinguish between three types of methodological bias as related to clinical trials for $\mathrm{AN}$, and consider their cumulative impact upon the existing evidence base for AN. Protocol registration bias refers to the nonprospective registration of trial protocols in ClinicalTrials.gov prior to the listed project start date, meaning that trials were registered after data collection or preliminary analyses had commenced. Reporting bias refers to the non-publication of study findings. Replication bias simply refers to the non-replication of findings from individual studies, anywhere in the published literature.

\section{Method}

We screened clinical trials listed on ClinicalTrials.gov, the world's largest open-access repository of federally- and privately-funded biomedical and healthrelated clinical trials, operated by the National Institutes of Health in the United States. Despite being operationally based in the United States, eligible trials from across the globe may be registered. Trial registrations on ClinicalTrials.gov typically 
permit open access to trial protocols, hypotheses, outcome measures, and within one year of study completion, summary results.

\section{Search Criteria and Data Extraction}

A key word search for 'anorexia nervosa' was performed on the ClinicalTrials.gov registry for AN trials registered between January $1^{\text {st }}, 2000$ and December $3^{\text {rd }}$, 2018. Trials of any methodological design were included, provided that they (i) focused on AN, and (ii) were not listed as “active” or "still recruiting”. Trials not relating to AN were excluded. Data extraction was undertaken independently by three members of the research team (SBM, EJC, JMN), and focused on (i) the status of studies as listed, (ii) the determination of whether results had been published or shared on ClinicalTrials.gov, (iii) the determination of whether studies were -reregistered, and (iv) whether results had been replicated. Publications stemming from registered trials were identified via ClinicalTrials.gov identifier numbers and attempts to identify replications of published findings were undertaken via exhaustive PubMed search in tandem with the identification of studies citing each eligible study. Given the non-ambiguity of these data, and their publicly available nature, there were no instances of inter-rater discordance. The data that support the findings of this study are openly available at https://osf.io/3yqv7/.

\section{Results}

This article is protected by copyright. All rights reserved. 
In total, 201 trials were identified, spanning trials in 21 different countries and 3 continents. Of these, 63 were currently active and still recruiting, while 37 were listed as either terminated $(N=8)$, withdrawn $(N=5)$ or of unknown status $(N=24)$. Of the trials listed as terminated or withdrawn, no summary results were shared from a single study. Of the remaining 101 trials listed as completed, two were excluded due to a central focus on anorexia cachexia rather than anorexia nervosa. Figure 1 illustrates a flow chart of study results.

Of these 99 eligible trials listed as completed, only 25 (25.25\%) reported summary results on ClinicalTrials.gov. Overall, only 41 trials (41.4\%) were published in peer-reviewed manuscripts, and a further 10 trials (10.1\%) reported raw summary data on ClinicalTrials.gov without publishing these findings in peer-reviewed publications. Interestingly, of the 10 trials which reported raw summary data on ClinicalTrials.gov and did not publish findings in peer-reviewed journals, 9 trials (90\%) demonstrated raw summary evidence which failed to support primary hypotheses. Overall, 48 trials (48.5\%) listed as completed did not report findings on ClinicalTrials.gov or in published manuscripts.

\section{Registration}

Of the 41 trials with findings published in peer reviewed manuscripts, only 8 (19.5\%) demonstrated evidence of prospective trial registration. Of these 8 prospective trial registrations, however, only 1 clearly articulated hypotheses and primary outcome measures. The remaining 33 trials (80.5\%) did not demonstrate evidence of prospective trial registration. Lastly, of the 41 trials with findings 
reported in the published literature, only 7 trials (17\%) have had their broad conceptual findings replicated in other datasets. Findings from the remaining 34 published trials (83\%) have not been replicated by other research groups. Moreover, across all studies, no trials shared de-identified open access to raw datasets or analytic scripts, both of which have been outlined as key components of reproducible science (Munafò et al., 2017). Figure 2 illustrates the cumulative impact of methodological bias among these trials.

\section{Discussion}

Our assessment of the current evidence base for AN suggests a pervasive cumulative impact of methodological bias. Among completed clinical trials, only a quarter reported summary results on ClinicalTrials.gov and fewer than half of all completed trials reported their findings on ClinicalTrials.gov or in peer-reviewed scientific journals. Among the trials that disseminated their findings in peer-reviewed manuscripts, fewer than one fifth demonstrated evidence of prospective clinical trial registration, or have had their primary findings replicated. This represents less than $10 \%$ of all completed registered trials, and less than $5 \%$ of all registered trials overall.

Until recently, few systematic incentives have been in place to encourage researchers to undertake the often time-consuming pre-registration of clinical trials. The low rates of pre-registration among AN trials accords with analyses of RCTs in broader areas of psychiatry and clinical psychology (Cybulski et al., 2016), although the profound underfunding of eating disorder research relative to other 
psychopathologies (Murray et al., 2017) may additionally impact the extent to which researchers can undertake the administrative burden of data sharing and prospective trial registration protocols. Funding agencies should consider providing additional resources for researchers to comprehensively address the calls for more transparent research.

Rules and regulations have now been advocated at a systems levels to prevent bias. For instance, since 2005 the ICMJE has required prospective registration of clinical trials as a prerequisite for publication; however, many individual journals do not require registration and others allow for retrospective registration (Harriman \& Patel 2016). Moreover, an increasing number of journals, including the International Journal of Eating Disorders, now support Registered Reports, where editorial decisions to publish research studies are based on study rationale and methodology, prior to data collection, which may alleviate potential researcher biases in confirming study hypotheses. The creation of centralized trial registration reporting repositories (i.e., ClinicalTrials.gov) facilitates standardization in reporting, and the non-reporting of transparent summary data may result in fines and sanctions for both researchers and their institutions. However, despite these rules and regulations, we found that only a minority of trials on AN published in peer-reviewed journals were prospectively registered.

We also found that less than a quarter of all registered clinical trials were published in peer-reviewed literature. The non-publication of completed trials in particular is an important issue, and is potentially impacted by bias around study 
results (i.e., the file drawer problem). One potential solution could be the adoption of Registered Reports in which peer-review and editorial decision making are undertaken prior to the collection of data, and is thus uninfluenced by results. This, in turn, may reduce the pressure on researchers to report 'positive' results in order to have their work published.

A particularly pernicious trend in clinical research may relate to the nonreplication of study findings. In the pursuit of novel findings, it is possible that replication studies are deemed less worthy of publication, by both researchers and editors alike. Moreover, funding for replication efforts appears scant, as the focus of funding agencies has shifted towards innovative and mechanistic discoveries. As such, an emphasis on the replication of study findings is a crucial priority for trials relating to AN.

Importantly, other clinical trial registries exist. While our study focused on only one, albeit the largest international clinical trial registry, it is important to note that not every clinical trial for AN is included in the present study. Further, while the FDA requires prospective registration of most drug trials, the same law does not apply to psychosocial interventions (de Vries et al, 2018). As such, the extent to which these trends apply broadly is uncertain. An important step forward in this line of inquiry relates to the replication of the current study across other trial registries and psychopathologies, and the further investigation of potential barriers to study publication and the dissemination of results. In particular, it is important to investigate whether non-published findings have been submitted for peer-review, 
Notwithstanding, we found evidence of pervasive reporting bias among clinical trials for AN, which likely compounds the methodological bias identified in recent meta-analyses (Murray et al., 2019; Zeeck et al., 2018). Cumulatively, these biases may impact the practice of evidence-based treatment. Indeed, clinical decision making, broader policy decision making, and the construction of research priorities are all inherently informed by the peer-reviewed evidence base, and the cumulative effects of methodological bias are potentially far reaching. Clinicians and researchers should be aware of these biases. 
Registration, Reporting, and Replication in Anorexia Nervosa

\section{Acknowledgements}

SBM is supported by the National Institute of Mental Health (K23 MH115184). EJC is supported by the Fulbright Commission and Argentine Ministry of Education. DSQ is supported by the Novo Nordisk Foundation (NNF160C0019856). SG is supported by the Australian National Health and Medical Research Council (1121538). JMN is supported by the Pediatric Scientist Development Program (NIH K12HD000850-33) and the American Heart Association ().

Declaration of Interest: SBM reports royalties from Routledge and Oxford University Press.

This article is protected by copyright. All rights reserved. 
Registration, Reporting, and Replication in Anorexia Nervosa

\section{References}

Anderson, M.L., Chiswell, K., Peterson, E.D., Tasneem, A., Topping, J., \& Califf, R.M. (2015). Compliance with results reporting at ClincalTrials.gov. New England Journal of Medicine, 372, 1031-1039.

Baker, M. (2016). 1,500 scientists lift the lid on reproducibility. Nature, 533, 452-454.

Camerer, C.F., Dreber, A., Holzmeister, F., Ho, T.H., Huber, J., ... Wu, H. (2019). replicability of social science experiments in Nature and Science between 2010 and 2015. Nature Human Behaviour, 2, 637-644.

Cybulski, L., Mayo-Wilson, E., \& Grant, S. (1026). Improving transparency and reproducibility through registration: The status of intervention trials published

This article is protected by copyright. All rights reserved. 
in clinical psychology journals. Journal of Consulting and Clinical Psychology, 84, 753-767.

De Angelis, C., Drazen, J.M., Frizelle, F.A., Haug, C., Hoey, J., ... Van Der Weyden M.B. (2004). Clinical Trial Registration: A Statement from the International Committee of Medical Journal Editors. New England Journal of Medicine, 351, 1250-1251.

De Vries, Y.A., Roest, A.M., de Jonge, P., Cuijpers, P., Munafò, M.R., \& Bastiaansen, J.A. (2018). The cumulative effect of reporting and citation biases on the apparent efficacy of treatments: The case of depression. Psychological Medicine,48, 2453-2455.

Fichter, M.M., Quadflieg, N., Crosby, R.D., \& Koch, S. (2017). Long-term outcome of anorexia nervosa: Results from a large clinical longitudinal study. International Journal of Eating Disorders, 50, 1018-1030.

Harriman, S.L., \& Patel, J. (2016). When are clinical trials registered? An analysis of prospective versus retrospective registration. Trials, 17, 187.

Mathieu, S., Boutron, I., Moher, D., Altman, D. G., \& Ravaud, P. (2009). Comparison of registered and published primary outcomes in randomized controlled trials. JAMA, 302, 977-984.

Murray, S.B., Pila, E., Griffiths, S., \& Le Grange, D. (2017). When illness severity and research dollars do not align: Are we overlooking eating disorders? World Psychiatry, 16, 321.

This article is protected by copyright. All rights reserved. 
Murray, S.B., Quintana, D.S., Loeb, K.L., Griffiths, S., \& Le Grange, D. (2019).

Treatment outcomes for anorexia nervosa: A systematic review and metaanalysis of randomized controlled trials. Psychological Medicine, 49, 535-544.

Munafo, M.R., Nosek, B.A., Bishop, D.V.M., Button, K.S., Chambers, C.D., ... Ioannidis, J.P.A. (2017). A manifesto for reproducible science. Nature Human Behaviour, 1: 0021.

Scott, A., Rucklidge, J.J., \& Mulder, R.T. (2015). Is Mandatory Prospective Trial Registration Working to Prevent Publication of Unregistered Trials and Selective Outcome Reporting? An Observational Study of Five Psychiatry Journals That Mandate Prospective Clinical Trial Registration. PLoS One, 10, e0133718.

Silbersahn, R., Uhlmann, E.L., Martin, D.P., Anselmi, P., Aust., ... Nosek, B.A. (2018). Many analysts, one data set: Making transparent how. Variations in analytic choices affect results. Advances in Methods and Practices in Psychological Science, 1, 337-356.

Turner, E.H., Matthews, A.M., Linardatos, E., Tell, R.A., \& Rosenthal, R. (2008). Selective publication of antidepressant trials and its influence on apparent efficacy. New England Journal of Medicine, 358, 252-60.

Walsh, C.G., Xia, W., Li, M., Denny, J.C., Harris, P.A., \& Malin, B.A. (2018) Enabling open-science initiatives in clinical psychology and psychiatry without sacrificing patients’ privacy: Current practices and future challenges. Advances in Methods and Practices in Psychological Science, 1, 104-114. 
Watson, H.J., \& Bulik, C.M. (2013). Update on the treatment of anorexia nervosa: Review of clinical trials, practice guidelines and emerging interventions. Psychological Medicine, 43, 2477-2500.

Zeeck, A., Herpertz-Dahlmann, B., Freiderich, H.C., Brockmeyer, T., Resmark, G., ... Hartmann, A. (2018). Psychotherapeutic treatment for anorexia nervosa: A systematic review and network meta-analysis. Frontiers in Psychiatry, 9, 158.

Figure 1: A flowchart of study results

This article is protected by copyright. All rights reserved. 


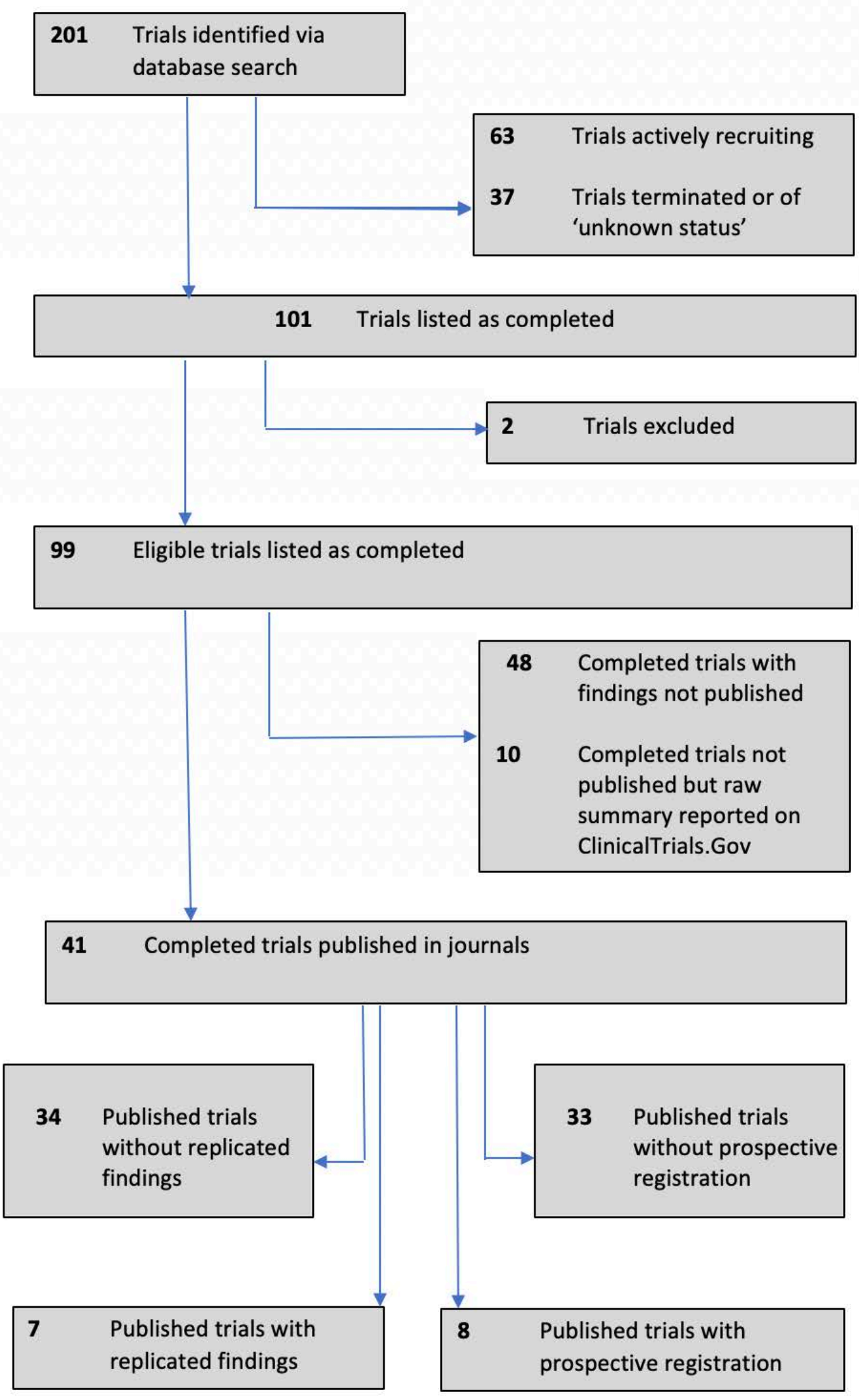

This article is protected by copyright. All rights reserved. 
Figure 2: An illustration of the cumulative effect of methodological bias in clinical trials for anorexia nervosa

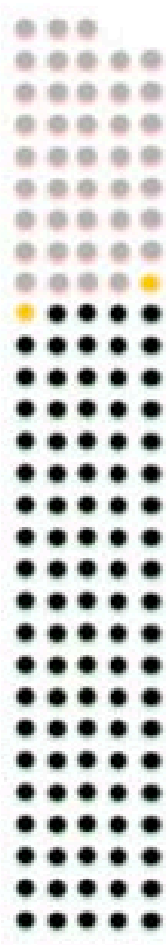

Trial Status

Completed Trials

Excluded Trials

Withdrawn/Terminated

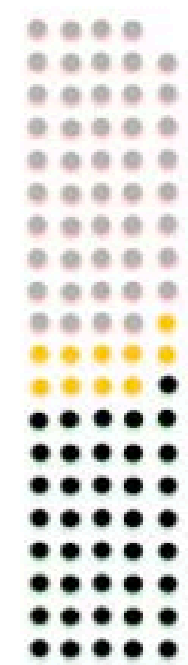

\section{Publication Status}

Findings are published

Raw summary data only

Findings not published

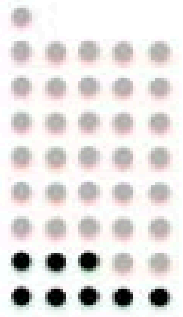

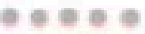

우응

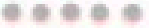

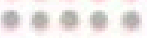

e

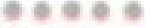

$\bullet \bullet \bullet \bullet \bullet$

\section{Registration Status}

Prospective registration

Post hoc registration

\section{Replication Status}

Findings replicated

Findings not replicated

This article is protected by copyright. All rights reserved. 


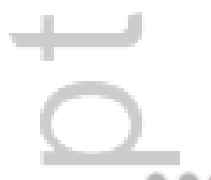

- 든

으른

- 으응

- 으응

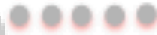

reva

으을

c neteot

$\bullet \bullet \bullet$

$\bullet \bullet \bullet \bullet$

- 0

부은

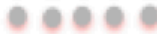

$\bullet \bullet \bullet \bullet$

으응

$0+0$

$-0 \circ \mathrm{c}$

- 응

$-60$

0

- 두은

으응

$\circ \circ$

$\bullet \bullet \bullet \bullet \bullet$

re

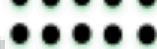

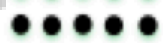

$\bullet \bullet \bullet \bullet$

$\bullet \bullet \bullet \bullet \bullet$

$\bullet \bullet \bullet \bullet$

$\bullet \bullet \bullet \bullet$

$\bullet \bullet \bullet \bullet \bullet$

$\bullet \bullet \bullet \bullet$

$\bullet \bullet \bullet \bullet$

$\circ \bullet$

e e e e

e e e

- 수응

으의

- 두을

e e e

$\circ \circ$

$\circ \div$

e

-

누의

- 응

- 0

우은

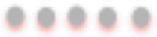

$\bullet \bullet \bullet \bullet \bullet$

$\bullet \bullet \bullet \bullet \bullet$

\section{Publication Status}

- Findings are published

- Raw summary data only

- Findings not published

\section{Registration Status}

- Prospective protocol registration

Post has protocol registration $\bullet \bullet \bullet \bullet$

\section{Replication Status}

- Results have been replicated

Results have not been replicated

EAT_23187_Screen Shot 2019-09-30 at 4.00.03 PM.png

This article is protected by copyright. All rights reserved. 


\section{University Library}

\section{- M M N E R VA A gateway to Melbourne's research publications}

Minerva Access is the Institutional Repository of The University of Melbourne

Author/s:

Murray, SB;Compte, EJ;Quintana, DS;Mitchison, D;Griffiths, S;Nagata, JM

Title:

Registration, reporting, and replication in clinical trials: The case of anorexia nervosa

Date:

2019-10-22

Citation:

Murray, S. B., Compte, E. J., Quintana, D. S., Mitchison, D., Griffiths, S. \& Nagata, J. M.

(2019). Registration, reporting, and replication in clinical trials: The case of anorexia nervosa. INTERNATIONAL JOURNAL OF EATING DISORDERS, 53 (1), pp.138-142. https:// doi.org/10.1002/eat.23187.

Persistent Link:

http://hdl.handle.net/11343/286525 